THE AsTROPHYSICAL JOURNAL, 544:43-48, 2000 November 20

(c) 2000. The American Astronomical Society. All rights reserved. Printed in U.S.A.

\title{
REIONIZATION CONSTRAINTS ON THE CONTRIBUTION OF PRIMORDIAL COMPACT OBJECTS TO DARK MATTER
}

\author{
M. Coleman Miller \\ Department of Astronomy, University of Maryland, College Park, MD 20742-2421; miller@astro.umd.edu \\ Received 2000 March 13; accepted 2000 June 27
}

\begin{abstract}
Many lines of evidence suggest that nonbaryonic dark matter constitutes $\sim 30 \%$ of the critical closure density, but the composition of this dark matter is unknown. One class of candidates for the dark matter is compact objects formed in the early universe, with typical masses $M \sim 0.1-1 M_{\odot}$, to correspond to the mass scale of objects found with microlensing observing projects. Specific candidates of this type include black holes formed at the epoch of the QCD phase transition, quark stars, and boson stars. Here we show that accretion onto these objects produces substantial ionization in the early universe, with an optical depth to Thomson scattering out to $z \sim 1100$ of $\tau \approx 2-4\left[f_{\mathrm{CO}} \epsilon_{-1}\left(M / M_{\odot}\right)\right]^{1 / 2}\left(H_{0} / 65\right)^{-1}$, where $\epsilon_{-1}$ is the accretion efficiency $\epsilon \equiv L / \dot{M} c^{2}$ divided by 0.1 , and $f_{\mathrm{CO}}$ is the fraction of matter in the compact objects. The current upper limit to the scattering optical depth, based on the anisotropy of the microwave background, is $\approx 0.4$. Therefore, if accretion onto these objects is relatively efficient, they cannot be the main component of nonbaryonic dark matter.
\end{abstract}

Subject headings: accretion, accretion disks - cosmic microwave background - cosmology: theory early universe

\section{INTRODUCTION}

Observations of the rotation curves of galaxies and clusters, in addition to joint fits of Type Ia supernova data and the power spectrum of the cosmic microwave background, suggest that the density of matter in the current universe is $\sim 30 \%$ of the closure density, i.e., $\Omega_{m} \sim 0.3$. However, the success of big bang nucleosynthesis in explaining the primordial abundances of light elements, especially the primordial abundance ratio of $\mathrm{D} / \mathrm{H}$, requires that the contribution of baryons is only $\Omega_{b} h^{2}=0.019 \pm 0.0024$ (95\% confidence; Tytler et al. 2000), where $h \equiv H_{0} / 100 \mathrm{~km}$ $\mathrm{s}^{-1} \mathrm{Mpc}^{-1}$ and $H_{0}$ is the present-day Hubble constant. The majority of the matter must be something else.

One class of possibilities involves hypothesized exotic particles, from light particles such as axions (Peccei \& Quinn 1977) to heavier particles such as the neutralino (e.g., Jungman, Kamionkowski, \& Greist 1996), or even ultramassive particles such as "WIMPzillas" (Kolb, Chung, \& Riotto 1999; Hui \& Stewart 1999). Another class, which we focus on in this paper, involves dark matter that occurs primarily in $\sim 0.1-1 M_{\odot}$ clumps. This class, which has received recent attention because this is the mass scale of objects discovered by microlensing projects such as MACHO, EROS, and OGLE, has several specific candidates. For example, black holes may have formed during the QCD phase transition from quark matter to nucleonic matter (Jedamzik 1997, 1998; Niemeyer \& Jedamzik 1999; note, however, that the perturbation spectrum must be peaked and finely tuned, see Schwarz, Schmid, \& Widerin 1997), during which the horizon mass was plausibly in the 0.1-1 $M_{\odot}$ range. Other suggestions involve quark stars (Banerjee et al. 2000), boson stars (Colpi, Shapiro, \& Wasserman 1986; Mielke \& Schunck 2000), and stars formed of mirror matter (Mohapatra \& Teplitz 1999). Here we consider those members of this class that involve primordial compact objects, specifically those objects that (1) existed before the $z \sim 1100$ epoch of decoupling, and (2) have a mass-to-radius ratio of $G M / R c^{2} \gtrsim 0.1$. These include black holes, quark stars, and boson stars, but not mirror matter stars, since they are envisioned to form at comparatively late times and to be comparable to ordinary stars in their compactness (Mohapatra \& Teplitz 1999).

Primordial compact objects will accrete from the ambient medium and will therefore generate substantial luminosity. This luminosity can ionize the surrounding medium. Unlike the energy spectra from ordinary stars, which drop off rapidly above the ground-state ionization energy of hydrogen, the energy spectra from accreting compact objects are known observationally to be very hard, with substantial components above $1 \mathrm{keV}$ and often extending above 100 $\mathrm{keV}$. An important consequence of this is that whereas the Stromgren sphere of ionization around, say, an O or B star is extremely sharply defined, with an exponentially decreasing ionization fraction outside the critical radius, the ionization fraction produced by an accreting compact object dies off relatively slowly with radius, as $r^{-3 / 2}$ (Silk 1971; Carr 1981). Therefore, accretion onto a primordial object can produce ionization over a large volume in the early universe. If the resulting optical depth to Thomson scattering is too large, it will conflict with the upper limit to this optical depth derived from the observed anisotropy of the microwave background (Griffiths, Barbosa, \& Liddle 1999). Conversely, the upper limit on the optical depth can be used to constrain the properties of primordial compact objects, if these are proposed as the dominant component of dark matter.

Here we calculate the ionization produced by compact objects accreting in the early universe. We find that the ionization produced by secondary electrons, an effect not included in previous analyses of reionization by accretion, substantially increases the ionization fraction and hence the optical depth to Thomson scattering. In $\S 2$ we show that the Thomson optical depth out to the $z \approx 1100$ redshift of decoupling is $\tau \approx 2-4\left[f_{\mathrm{CO}} \epsilon_{-1}\left(M / M_{\odot}\right)\right]^{1 / 2}\left(H_{0} / 65\right)^{-1}$, where $\epsilon_{-1}$ is the accretion efficiency, $L / \dot{M} c^{2}$, divided by 0.1 , and $f_{\mathrm{CO}}$ is the fraction of matter in primordial compact objects. We compare this result to the current observational upper limit of $\tau<0.4$, and show that either low accretion 
efficiency or low mass is required if dark matter is mostly composed of primordial compact objects. In $\S 3$ we consider low-efficiency accretion, such as flows dominated by advection or wind outflow. We show that the constraints from ionization are especially tight on objects without horizons. In $\S 4$ we place this result in the context of previous constraints on, for example, primordial black holes as the main component of dark matter. We also discuss future improvements to our result. In particular, we show that the expected accuracy of optical depth measurements with the Microwave Anisotropy Probe (MAP) and Planck could decrease the upper bound on $f_{\mathrm{CO}} \epsilon_{-1}\left(M / M_{\odot}\right)$ by a further factor of $\sim 100$.

\section{CALCULATION OF OPTICAL DEPTH}

If the number density of baryons is $n(z) \approx z^{3} n_{0}$ (where in this entire calculation we assume $z \gg 1$ ) and the ionization fraction is $x(z)$, then the optical depth to Thomson scattering between redshifts $z$ and $z+d z$ is

$$
d \tau(z)=n(z) x(z) \sigma_{\mathrm{T}} d s(z),
$$

where $\sigma_{\mathrm{T}}=6.65 \times 10^{-25} \mathrm{~cm}^{2}$ is the Thomson scattering cross section, and

$$
d s(z)=\frac{1}{H_{0}} \frac{c d z}{(1+z) E(z)}
$$

is the distance traveled by a photon in this redshift interval. Here $E(z)=\left[\Omega_{m}(1+z)^{3}+\Omega_{R}(1+z)^{2}+\Omega_{\Lambda}\right]^{1 / 2}$, and $\Omega_{m}$, $\Omega_{R}$, and $\Omega_{\Lambda}$ are the current contributions to the mass energy of the universe from, respectively, matter, curvature, and the cosmological constant. At $z \gg 1$ the first term dominates, so that $E(z) \approx \Omega_{m}^{1 / 2} z^{3 / 2}$ and $d s(z) \approx c H_{0}^{-1} \Omega_{m}^{-1 / 2} z^{-5 / 2} d z$. The differential optical depth is then

$$
d \tau(z) \approx n_{0} z^{3} x(z) \sigma_{\mathrm{T}} d s(z)=n_{0} x(z) \sigma_{\mathrm{T}} \frac{c}{H_{0} \Omega_{m}^{1 / 2}} z^{1 / 2} d z
$$

(see also Haiman \& Knox 1999). This needs to be integrated out to the $z \sim 1100$ redshift of decoupling to determine the optical depth to scattering in the early universe. The main unknown in this expression is the ionization fraction, $x(z)$. In the remainder of this section, therefore, we compute the ionization produced by radiation from accreting compact objects. In $\S 2.1$ we compute the luminosity and spectrum of this radiation. We assume Bondi-Hoyle accretion and a spectrum corresponding to that observed from many neutron stars and black hole candidates. In $\S 2.2$ we use the ionization balance equation to calculate the ionization produced by a single source. We include the effects of ionization by secondary electrons, which is a significant effect not included in the analysis of pregalactic black hole accretion by Carr (1981). In $\S 2.3$ we show that the ionizing flux from sources spread throughout the universe significantly increases the ionization fraction. Finally, in $\S 2.4$ we calculate the optical depth to Thomson scattering out to the $z \sim 1100$ redshift of decoupling, including the effects of Compton cooling by the microwave background.

\subsection{Luminosity and Spectrum of Radiation}

Let us now consider accreting objects of mass $M$. Suppose that these masses are moving with the Hubble flow, so that the main parameter governing the accretion rate is the speed of sound in the gas at infinity, $a_{\infty}=$ $\left(\Gamma_{1} k T / \mu m_{p}\right)^{1 / 2}$, where $\Gamma_{1}$ is the polytropic index, $m_{p}$ is the mass of the proton, and $\mu$ is the mean molecular weight. For pure hydrogen $(\mu=1 / 2)$, the mass accretion rate from a perfect gas with $\Gamma_{1}=5 / 3$ is then

$$
\dot{M}=1.2 \times 10^{10}\left(\frac{M}{M_{\odot}}\right)^{2} \frac{\rho_{\infty}}{10^{-24} \mathrm{~g} \mathrm{~cm}^{-3}} T_{4}^{-3 / 2} \mathrm{~g} \mathrm{~s}^{-1},
$$

where $T_{4} \equiv T_{\infty} / 10^{4} \mathrm{~K}$ and $T_{\infty}$ is the temperature of the gas at infinity. For a primordial composition of $75 \%$ hydrogen and $25 \%$ helium by mass, this accretion rate is more than doubled, because helium has half the velocity of hydrogen for a given temperature, and hence accretes at 8 times the rate for a given mass density. We therefore take the coefficient to be $3 \times 10^{10}$. If accretion produces a luminosity with an efficiency $0.1 \epsilon_{-1}$, so that $L=10^{20} \epsilon_{-1} \dot{M} \mathrm{ergs} \mathrm{s}^{-1}$, then

$$
L=3 \times 10^{30}\left(\frac{M}{M_{\odot}}\right)^{2} \frac{\rho_{\infty}}{10^{-24} \mathrm{~g} \mathrm{~cm}^{-3}} T_{4}^{-3 / 2} \mathrm{erg} \mathrm{s}^{-1} .
$$

The best estimate of the baryon density in the current universe from big bang nucleosynthesis constraints (Tytler et al. 2000) is

$$
\rho_{B_{0}}=3.6 \pm 0.4 \times 10^{-31} \mathrm{~g} \mathrm{~cm}^{-3} .
$$

At a redshift $z$, this density is therefore $(1+z)^{3} \rho_{B_{0}} \approx z^{3} \rho_{B_{0}}$. Hence, if the compact object accretes matter with the average baryonic density in the universe, the luminosity at redshift $z$ is

$$
L \approx 10^{24} z^{3}\left(\frac{M}{M_{\odot}}\right)^{2} T_{4}^{-3 / 2} \operatorname{ergs~s}^{-1} .
$$

Pressure balance of a hot $\mathrm{H}$ II region with the cooler exterior universe may decrease the density of accreting matter and therefore decrease this luminosity (see below). In accreting black hole sources from stellar mass to active galactic nuclei (AGNs), and also in some accreting neutron stars, the spectrum often has a power-law tail with a photon number index that ranges between $\sim 1.5$ and 3 up to some $E_{\max }$, depending on the spectral state (see, e.g., Grove et al. 1998). This implies a differential luminosity spectrum $d L(E) / d E \propto E^{-\alpha} \exp \left(-E / E_{\max }\right)$, with $\alpha=0.5-2$. The results of our calculation are fairly insensitive to the assumed spectrum; for simplicity, we will assume $\alpha=1$, i.e., a spectrum with equal power in equal logarithmic intervals, and will later state results for $\alpha=0$ and $\alpha=2$. Normalizing this spectrum so that the total luminosity above $E_{0}=13.6 \mathrm{eV}$ is $L$, the differential photon flux at energy $E$ a distance $R$ from the compact object is

$$
F(E)=e^{-\tau(E)} \frac{d L / d E}{4 \pi R^{2} E}=e^{-\tau(E)} \frac{L}{4 \pi \ln \left(E_{\max } / E_{0}\right) E^{2} R^{2}} e^{-E / E_{\max }} .
$$

Here $\tau(E)$ is the optical depth at a distance $R$ from the source to photons of energy $E$. Note that Carr (1981) chose a spectrum of a bremsstrahlung form $\left(d L / d E \propto e^{-E / E_{\max }}\right)$, and hence had a different energy dependence and normalization for the photon number flux.

\subsection{Secondary Ionization and Ionization Balance}

A given photon can effectively produce many ionizations, because the ionized electrons can collisionally ionize other atoms (see, e.g., Silk \& Werner 1969; Silk 1971). The collisional cross section exceeds $10^{-17} \mathrm{~cm}^{2}$ for electron ener- 
gies between $\sim 15 \mathrm{eV}$ and $1 \mathrm{keV}$ (see Dalgarno, Yan, \& Liu 1999 for a recent discussion of electron energy deposition). Dalgarno et al. (1999) calculate that the mean energy per ion pair decreases with increasing initial electron energy, reaching a limit of $36.1 \mathrm{eV}$ per pair at energies greater than $200 \mathrm{eV}$. Using their Figure 6, we adopt an approximate value of $E / 3 E_{0}$ hydrogen atoms ionized by a photon of initial energy $E$; this is a rough average over the energy range of interest, and we assume for simplicity that it is constant over that range. Note that the assumed factor of 3 has little impact on the calculated ionization fraction $x$, because for $x \ll 1$ the ionization fraction scales as the square root of the ionization rate.

The effective ionization rate produced by the photons generated by accretion is therefore (adapting the formula of Carr 1981)

$$
\zeta_{\mathrm{H}} \approx \int_{E_{0}}^{\infty} \sigma_{1}\left(\frac{E}{E_{0}}\right)^{-3} \frac{E}{3 E_{0}} F(E) d E,
$$

where $\sigma_{1} \approx 2 \times 10^{-17} \mathrm{~cm}^{2}$ is the abundance-weighted ionization cross section at $E_{0}$. The integrand in this formula is a constant factor

$$
\frac{E_{\max }}{3 E_{0} \ln \left(E_{\max } / E_{0}\right)}
$$

times the integrand in the corresponding formula in Carr (1981). The difference arises because we assume a different form for the spectrum and account for the ionization produced by secondary electrons. The remainder of the analysis of the ionization region created by a single source follows the treatment of Carr (1981), with this factor included. This is a large factor, of the order of 25 for $E_{\max }=10^{-8} \mathrm{ergs}$, and it therefore makes a crucial difference to the overall ionization. If $E_{\max } \gg E_{0}$, the ionization rate is approximately (Silk et al. 1972)

$$
\zeta_{\mathrm{H}} \approx \frac{E_{\max }}{3 E_{0} \ln \left(E_{\max } / E_{0}\right)} \frac{\sigma_{1} L}{12 \pi E_{\max } R^{2}} \frac{1-\exp \left(-\tau_{0}\right)}{\tau_{0}},
$$

where

$$
\tau_{0}=\int_{0}^{R} n_{\mathrm{H}}(1-x) \sigma_{1} d R,
$$

and the first factor indicates the correction factor to the expression of Carr (1981). Here $x$ is the ionized fraction at radius $R$.

The ionization balance equation is

$$
\alpha n_{\mathrm{H}}^{2} x^{2}=\zeta_{\mathrm{H}} n_{\mathrm{H}}(1-x),
$$

where around $T \approx 10^{4} \mathrm{~K}$, the recombination coefficient not including single-photon transitions to the ground state (which would release ionizing photons) is $\alpha \approx 2.6$ $\times 10^{-13} T_{4}^{-0.75} \mathrm{~cm}^{3} \mathrm{~s}^{-1}$ (Hummer 1994). Far from the accreting compact object, where $x \ll 1$ and $\tau_{0} \gg 1$, the ionization fraction is

$$
x=\left[\frac{E_{\max }}{3 E_{0} \ln \left(E_{\max } / E_{0}\right)}\right]^{1 / 2} \frac{1}{\sqrt{8}}\left(\frac{R}{R_{s}}\right)^{-3 / 2},
$$

where

$$
R_{s} \equiv\left(\frac{2 L}{3 \pi \alpha n_{\mathrm{H}}^{2} E_{\mathrm{max}}}\right)^{1 / 3}
$$

Again, the initial factor in the equation for $x$ is the correction factor, which therefore increases the ionization fraction far from the compact object by a factor of $\sim 5$; note that the only remaining dependence on $E_{\max }$ is $\left[\ln \left(E_{\max } / E_{0}\right)\right]^{-1 / 2}$, so this result is very insensitive to the high-energy cutoff of the spectrum.

\subsection{Contribution of Multiple Sources}

The total ionization rate, $\zeta_{\mathrm{H}}$, must be summed over the contributions of all sources. At large distances from a source, $\tau_{0} \propto R \gg 1$, so that $\zeta_{\mathrm{H}} \sim R^{-3}$. For multiple sources separated by an average distance $R_{\text {sep }}$, the ionizing rate is larger than the single-source ionizing rate at a distance $R_{\text {sep }}$ by a factor

$$
\frac{\sum \zeta_{\mathrm{H}}}{\zeta_{\mathrm{H}}\left(r=R_{\mathrm{sep}}\right)}=\int_{R_{\mathrm{sep}}}^{R_{\max }}\left(\frac{r}{R_{\mathrm{sep}}}\right)^{-3} 4 \pi r^{2} n_{\mathrm{CO}} d r .
$$

Here $n_{\mathrm{CO}}=10^{-62} z^{3}\left(M / M_{\odot}\right)^{-1} \Omega_{\mathrm{CO}} \mathrm{cm}^{-3}$ is the number density of compact objects at redshift $z$, where $\Omega_{\mathrm{CO}}$ is the fraction of the closure density in compact objects. In addition, $R_{\max } \approx \min \left[10^{31} z^{-3} x^{-1}, c / H(z)\right] \mathrm{cm}$ is the mean free path to Thomson scattering. The separation distance is approximately given by $\left[(4 / 3) \pi R_{\text {sep }}^{3}\right]^{-1}=n_{\mathrm{CO}}$, so $R_{\text {sep }}^{3} \approx$ $[3 /(4 \pi)] n_{\mathrm{CO}}^{-1}$. Therefore,

$$
\frac{\sum \zeta_{\mathrm{H}}}{\zeta_{\mathrm{H}}\left(r=R_{\mathrm{sep}}\right)} \approx 3 \ln \left(\frac{R_{\mathrm{max}}}{R_{\mathrm{sep}}}\right) .
$$

The ratio of radii is typically $10^{6}-10^{8}$, so the enhancement due to the contributions of multiple sources is approximately a factor of 50 .

When multiple sources are included, the ionization fraction (for $x \ll 1$ ) is increased by a factor that is approximately the square root of the factor by which the ionization rate is enhanced. At a distance $R$, the rate is enhanced by a factor

$$
\zeta_{\mathrm{H}} \rightarrow \zeta_{\mathrm{H}}\left[1+50\left(\frac{R}{R_{\text {sep }}}\right)^{3}\right]
$$

and hence the ionization fraction including multiple sources is

$$
\begin{aligned}
x \approx & {\left[\frac{E_{\max }}{3 E_{0} \ln \left(E_{\max } / E_{0}\right)}\right]^{1 / 2} \frac{1}{\sqrt{8}}\left(\frac{R}{R_{s}}\right)^{-3 / 2} } \\
& \times\left[1+50\left(\frac{R}{R_{\text {sep }}}\right)^{3}\right]^{1 / 2} .
\end{aligned}
$$

Integrating this from $R_{s}$ to $R_{\text {sep }}$, the volume-averaged ionization is

$$
\bar{x} \approx 3\left[\frac{E_{\max }}{3 E_{0} \ln \left(E_{\max } / E_{0}\right)}\right]^{1 / 2}\left(\frac{R_{s}}{R_{\mathrm{sep}}}\right)^{3 / 2} .
$$

Here $R_{\text {sep }}=3 \times 10^{20} z^{-1}\left(M / M_{\odot}\right)^{1 / 3} \Omega_{\mathrm{CO}}^{-1 / 3} \mathrm{~cm}$. For comparison, if the spectrum has a differential luminosity slope $\alpha=0$, then the volume-averaged ionization is $\bar{x}(\alpha=0) \approx$ $\left(R_{\max } / R_{s}\right)^{1 / 6}\left(R_{s} / R_{\text {sep }}\right)^{3 / 2}$. This scales with $E_{\max }$ like $E_{\max }^{-4 / 9}$, and for a typical $E_{\max }=60 \mathrm{keV}$ is a factor of $\sim 2$ less than the average ionization for $\alpha=1$. If the spectrum has $\alpha=2$, as is observed for some black hole candidates in the soft state (Grove et al. 1998), then $\bar{x}(\alpha=2) \approx$ $5\left(E_{\max } / E_{0}\right)^{1 / 2}\left(R_{s} / R_{\text {sep }}\right)^{1 / 6}\left(R_{s} / R_{\text {sep }}\right)^{3 / 2}$. This is independent of $E_{\max }$ (there is an unwritten factor of order unity involving 


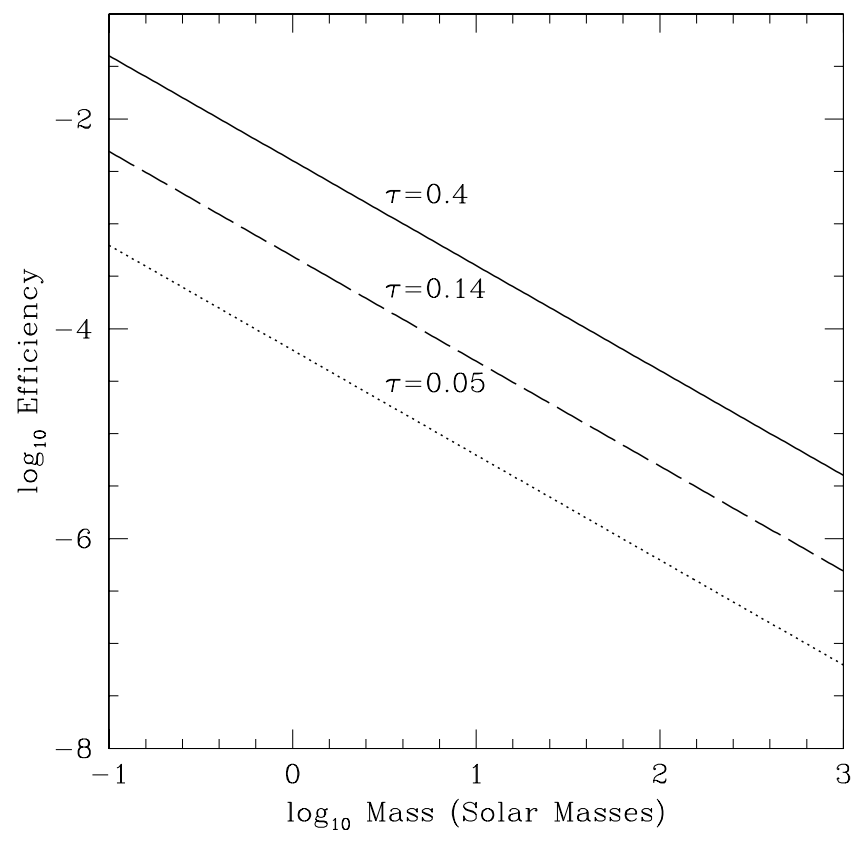

FIG. 1.-Limits on the accretion efficiency, $\epsilon=L / \dot{M} c^{2}$, of primordial compact objects as a function of their typical mass, if they are to make up the bulk of dark matter $\left(f_{\mathrm{CO}}=1\right)$. These limits assume no Rayleigh-Taylor mixing at the boundary of the $\mathrm{H}$ II region, and are therefore conservative. The three curves show the upper limits to the efficiency as a function of mass for the current upper limit to the Thomson optical depth of $\tau=0.4$ (solid curve) and future possible upper limits of $\tau=0.14$ (dashed curve) and $\tau=0.05$ (dotted curve).

$E_{\max }^{1 / 18}$ ), and for typical parameters is a factor of $\sim 2$ larger than $\bar{x}(\alpha=1)$. The average ionization is relatively independent of the spectral shape because there are compensating factors: a hard spectrum $(\alpha<1)$ has low ionization near the source, but its effects are substantial far from the source, whereas a soft spectrum $(\alpha>1)$ has higher ionization near the source but little effect far away.

\subsection{Optical Depth Including Compton Cooling and Pressure Balance}

An evaluation of this expression for the ionization fraction requires knowledge of the luminosity of individual sources and the average temperature of the matter in the universe. As pointed out by, e.g., Carr (1981), the dominant cooling process at high redshifts is inverse Compton cooling off the microwave background. If the temperature $T$ of the matter is much larger than the temperature $T_{r}$ of the radiation background, then the cooling rate per volume at redshift $z$ is

$$
\Gamma_{r} \approx 2 \times 10^{-38} x(z) z^{7} T_{4} \text { ergs } \mathrm{cm}^{-3} \mathrm{~s}^{-1} .
$$

The average heating rate is just the luminosity per source times the number density of sources:

$$
\Gamma_{h}=L n_{\mathrm{CO}}=10^{-38} \Omega_{\mathrm{CO}} z^{6} \text { ergs } \mathrm{cm}^{-3} \mathrm{~s}^{-1} .
$$

At $z \sim 1000$, where the optical depth to Thomson scattering exceeds unity and, as we will see, $x \sim 0.1$, the cooling rate dominates the heating rate, and hence the matter temperature is locked to the radiation temperature during this epoch (see also Carr 1981). This increases the recombination rate over most of the volume of interest, and therefore decreases the optical depth to scattering. Inside the $\mathrm{H}$ II region, by contrast, heating dominates cooling and the tem- perature remains close to $10^{4} \mathrm{~K}$ for $z>10$; in fact, Carr (1981) finds that the temperature is $T_{4}=\left(z / 10^{3}\right)^{0.3}$. The temperature difference means that pressure balance requires that the density inside the $\mathrm{H}$ II region be less than the average density by a factor of $\sim \bar{T} / T$; note, however, that this configuration requires the support against gravity of a denser by a less dense medium, which therefore is in principle Rayleigh-Taylor unstable. Hence, mixing could occur, which would decrease the temperature and increase the density of the $\mathrm{H}$ II region. If mixing does not occur, the density of the matter accreting onto the compact object is decreased by a factor of $\sim 0.27\left(z / 10^{3}\right)^{0.7}$. The ionization fraction and hence the optical depth would therefore be reduced by the square root of this factor, or about $0.5\left(z / 10^{3}\right)^{0.35}$. The uncertainty of whether there is an interchange instability and mixing thus produces an uncertainty of a factor of $\sim 2$ in the optical depth to scattering.

With these contributions, the average ionization is

$$
\bar{x}(z) \approx 5-10 \times 10^{-4} \epsilon_{-1}^{1 / 2}\left(\frac{M}{M_{\odot}}\right)^{1 / 2} \Omega_{\mathrm{CO}}^{1 / 2}\left[\frac{6}{\ln \left(E_{\max } / E_{0}\right)}\right]^{1 / 2} z^{0.7} .
$$

The differential optical depth to scattering is $d \tau(z)=$ $n_{0} x(z) \sigma_{\mathrm{T}}\left[c /\left(H_{0} \Omega_{m}^{1 / 2}\right)\right] z^{1 / 2} d z$, so using $n_{0}=2.2 \pm 0.3$ $\times 10^{-7} \mathrm{~cm}^{-3}$ and evaluating the constants, this is

$$
\begin{aligned}
d \tau(z) \approx & 1-2 \times 10^{-6}\left[\frac{6}{\ln \left(E_{\max } / E_{0}\right)}\right]^{1 / 2} \epsilon_{-1}^{1 / 2}\left(\frac{M}{M_{\odot}}\right)^{1 / 2} \\
& \times\left(\frac{H_{0}}{65 \mathrm{~km} \mathrm{~s}^{-1} \mathrm{Mpc}^{-1}}\right)^{-1} f_{\mathrm{CO}}^{1 / 2} z^{1.2} d z,
\end{aligned}
$$

where $f_{\mathrm{CO}} \equiv \Omega_{\mathrm{CO}} / \Omega_{m}$ is the fraction of matter in compact objects. Integrating from a small redshift to the redshift $z \approx 1100$ at decoupling finally gives

$$
\tau \approx 2-4\left[\frac{6 \epsilon_{-1}}{\ln \left(E_{\max } / E_{0}\right)} \frac{M}{M_{\odot}} f_{\mathrm{CO}}\right]^{1 / 2}\left(\frac{H_{0}}{65}\right)^{-1} .
$$

The effect of this optical depth on the observed cosmic microwave background (CMB) power spectrum is not identical to the effect of the same optical depth if it came from sudden and complete reionization at some lower redshift, $z \sim 10-40$. This is because the mechanism described here produces most of the optical depth at comparatively high redshifts, $z \gtrsim 800$, and hence for optical depths in excess of unity the scatterings occur close to recombination, where some of the primordial anisotropy is maintained. This would therefore mean that the last scattering would occur at lower redshift than commonly supposed, which would move the first Doppler peak in the CMB power spectrum to lower wavenumbers, as well as suppressing higher peaks. This is one possible explanation (see also Peebles, Seager, \& $\mathrm{Hu} 2000$ ) for the recent BOOMERANG nondetection of a significant second peak (deBernardis et al. 2000). In contrast, scattering at low redshift exponentially suppresses the primordial anisotropy at all scales. However, if the optical depth is less than unity this effect is less pronounced in the reionization mechanism discussed in this paper, because scatterings occur over a wide range of redshift and hence tend to smooth out small-scale anisotropies in the same way as would happen due to scattering at much lower redshifts. These qualitative effects are confirmed by simulations with CMBFAST (Seljak \& Zaldarriaga 1996 and sub- 
sequent papers), which show that for $\tau \lesssim 1$ the constraints on the optical depth from the observed CMB power spectrum are roughly the same for this mechanism as for late reionization.

The observational upper limit to $\tau$ from small-scale CMB anisotropy is $\tau \lesssim 0.4$ if $\Omega_{m}=0.3$ and the primordial power spectrum has an index $n=1$ (Griffiths et al. 1999). To be consistent with this limit, primordial compact objects must therefore be either low-efficiency accretors, low-mass objects, or a minor component of dark matter. If MACHOs lie in the Galactic halo (in the direction of the LMC they may instead be stars in the LMC itself; see Sahu 1994; Kerins \& Evans 1999), their mass spectrum has a peak in the $\sim 0.5-1 M_{\odot}$ range (Alcock et al. 2000). Note, however, that most of the mass in the halo is not in MACHOs (Gates et al. 1998; Alcock et al. 2000), and a higher mass component is not ruled out (Lasserre et al. 2000). Therefore, an explanation of these objects as a population that comprises most of the dark matter in the universe requires lowefficiency accretion, which we consider in the next section. These constraints are particularly strict for higher mass black holes. The joint limits on $M$ and $\epsilon$ are shown in Figure 1, for three different upper limits to the Thomson optical depth: $\tau=0.4,0.14$, or 0.05 , which are the optical depths obtained if the universe were fully and suddenly reionized at a redshift of $z_{\text {reion }}=40,20$, or 10 , respectively.

\section{EFFICIENCY OF ACCRETION}

In the last few years, there has been much discussion of the possibility that, for low accretion rates, an advectiondominated accretion flow (ADAF) is set up in which the radiative efficiency onto black holes is low because the matter flows almost radially into the hole, taking almost all its energy with it (e.g., Narayan \& Yi 1994; Abramowicz et al. 1995). The radiative efficiency, according to these solutions, could be in the range $\sim 10^{-3}-10^{-4}$ (see, e.g., Yi et al. 1996; the computed efficiency depends strongly on the emission mechanism), and hence might allow a large matter density in black holes. If the compact object does not have a horizon, then an ADAF will not reduce the radiative efficiency, so this is not a way out.

Another possibility is that most of the accreting matter does not reach the surface at all, perhaps because it is driven out in a wind. This is the basis of the advection-dominated inflow-outflow solution (ADIOS) proposed by Blandford \& Begelman (1999). Convective flow, in which the net inward flow rate at small radii is small, has also been found analytically by Quataert \& Narayan (1999) and in numerical simulations by Stone, Pringle, \& Begelman (1999). If in Bondi-Hoyle accretion the result of the accretion is inflow of a small fraction of matter combined with outflow of most of the matter, then the accretion efficiency onto even objects without horizons could be small. However, there is a crucial unsolved problem with these flows, which is whether they will remain at low efficiency indefinitely if there is a steady inflow of matter from infinity, as in Bondi-Hoyle accretion. If instead the accretion proceeds as in a dwarf nova, in which there is a long-term buildup of matter followed by a short-term, high-luminosity episode during which the accumulated matter is dumped onto the central object, then current accretion theory suggests that the accretion will generate radiation with an efficiency $\epsilon \sim 0.1$ regardless of the nature of the compact object. In such a case, neither black holes nor any other type of primordial compact object are viable candidates for most of the dark matter in the universe.

\section{DISCUSSION AND CONCLUSIONS}

Consideration of compact objects as components of dark matter has often been restricted to black holes, but many of the arguments apply more generally. Black holes with masses in excess of $\sim 10^{3} M_{\odot}$ are ruled out as a significant component of galactic halos because their dynamical interactions with globular clusters would destroy the clusters (for a recent calculation see Arras \& Wasserman 1999). The lack of an increase in the number of low equivalent width quasars with increasing redshift (expected to be caused by gravitational lensing) rules out a contribution $\Omega \gtrsim 0.1$ from any objects with masses between $\sim 10^{-2}$ and $20 M_{\odot}$ that are more compact than their Einstein radii (Dalcanton et al. 1994). The lack of observed lensing of cosmological gammaray bursts also allows weak limits to be placed on the contribution of black holes of various sizes: $\Omega<0.15$ at the $90 \%$ level for $M=10^{6.5} M_{\odot}, \Omega<0.9$ at the $1 \sigma$ level for $M=10^{-12.5}-10^{-9} M_{\odot}$, and $\Omega<0.1\left(z_{\mathrm{GRB}} \sim 1\right)$ or $\Omega<0.2$ $\left(z_{\text {GRB }} \sim 2\right)$ at the $95 \%$ level for $M=10^{-16}-10^{-13} M_{\odot}$ (Marani et al. 1999).

Here we show that ionization from compact-object accretion in the early post-decoupling universe is more significant than had been thought previously, because of the effects of secondary ionization by electrons. The result is that, barring inefficient accretion $\left(\epsilon<0.05\right.$ for $M=0.1 M_{\odot}$, $\epsilon<0.005$ for $\left.M=1 M_{\odot}\right)$, primordial compact objects in this mass range cannot make up a significant fraction of the mass of the universe, because they would ionize the universe enough to conflict with the measured small-scale anisotropies of the cosmic microwave background. If further analysis and numerical simulation of flows onto black holes demonstrates that the long-term time-averaged accretion efficiency is $\gtrsim 0.1$, as might happen if matter tends to pile up as in a dwarf nova and then accrete quickly with efficient radiation, then all masses greater than $\sim 0.1 M_{\odot}$ are excluded from making a significant contribution.

Future CMB missions such as $M A P$ and Planck could strengthen these constraints considerably. The optical depth resolution of $M A P$ is expected to be 0.022 , and that of Planck is expected to be 0.004 (Zaldarriaga, Spergel, \& Seljak 1997; Bouchet, Prunet, \& Sethi 1999; Eisenstein, Hu, $\&$ Tegmark 1999). Since the existence of a Ly $\alpha$ emitter at $z=5.64$ (see Haiman \& Spaans 1999) shows that reionization must have occurred before then, this means that both satellites, and especially Planck, will be able to detect the effects of ionization regardless of the actual redshift of reionization. If $z_{\text {reion }} \sim 10$, then the redshift of reionization could even be determined directly with SIRTF or NGST via, e.g., analysis of the damping wing of the Gunn-Peterson trough (Miralda-Escudé 1998) or detection of transmitted flux between Lyman resonances (Haiman \& Loeb 1999). The upper limit on the product $\epsilon_{-1}\left(M / M_{\odot}\right) f_{\text {CO }}$ scales as $\tau_{\text {scatt }}^{2}$ (or, for $z \gg 1$, as $z_{\text {reion }}^{3}$ ), so if $z_{\text {reion }} \sim 10$, this upper limit is decreased by almost a factor of 100. In this case, barring extremely inefficient accretion, dark matter must be composed of less compact objects or of WIMPs.

We thank Sylvain Veilleux, Andy Young, Jim Stone, Eve Ostriker, and Scott Dodelson for comments. This work was supported in part by NASA ATP grant NAG 5-9756. 


\section{REFERENCES}

Abramowicz, M., Chen, X.-M., Kato, S., Lasota, J.-P., \& Regev, O. 1995, ApJ, 438, L37

Alcock, C., et al. 2000, ApJ, 542, 281

Arras, P., \& Wasserman, I. 1999, MNRAS, 306, 257

Banerjee, S., Bhattacharya, A., Ghosh, S. K., Raha, S., \& Sinha, B. 2000, preprint (astro-ph/0002007)

Blandford, R. D., \& Begelman, M. C. 1999, MNRAS, 303, L1

Bouchet, F. R., Prunet, S., \& Sethi, S. K. 1999, MNRAS, 302, 663

Carr, B. J. 1981, MNRAS, 194, 639

Colpi, M., Shapiro, S. L., \& Wasserman, I. 1986, Phys. Rev. Lett., 57, 2485

Dalcanton, J. J., Canizares, C. R., Granados, A., Steidel, C. C., \& Stocke, J. T. 1994, ApJ, 424, 550

Dalgarno, A., Yan, M., \& Liu, W. 1999, ApJS, 125, 237

de Bernadis, P., et al. 2000, Nature, 404, 955

Eisenstein, D. J., Hu, W., \& Tegmark, M. 1999, ApJ, 518, 2

Gates, E. I., Gyuk, G., Holder, G. P.. \& Turner, M. S. 1998, ApJ, 500, L145

Griffiths, L. M., Barbosa, D., \& Liddle, A. R. 1999, MNRAS, 308, 854

Grove, J. E., Johnson, W. N., Kroeger, R. A., McNaron-Brown, K., Skibo, J. G., \& Phlips, B. F. 1998, ApJ, 500, 899

Haiman, Z., \& Knox, L. 1999, ASP Conf. Ser. 181, Microwave Foregrounds, ed. A. de Oliveira-Costa \& M. Tegmark (San Francisco: ASP), 227

Haiman, Z., \& Loeb, A. 1999, ApJ, 519, 479

Haiman, Z., \& Spaans, M. 1999, ApJ, 518, 138

Hui, L., \& Stewart, E. D. 1999, Phys. Rev. D, 60, 023518

Hummer, D. G. 1994, MNRAS, 268, 109

Jedamzik, K. 1997, Phys. Rev. D, 55, 5871 . 1998, Phys. Rep., 307, 155
Jungman, G., Kamionkowski, M., \& Greist, K. 1996, Phys. Rep., 267, 195

Kerins, E. J., \& Evans, N. W. 1999, ApJ, 517, 734

Kolb, E. W., Chung, D. J. H., \& Riotto, A. 1999, in Dark Matter in Astro and Particle Physics, Proc. DARK98, ed. H. V. Klapdor-Kleingrothaus \& L. Baudis (Philadelphia: Inst. Physics), 592

Lasserre, T., et al. 2000, A\&A Lett., 355, 39

Marani, G. F., Nemiroff, R. J., Norris, J. P., Hurley, K., \& Bonnell, J. T. 1999, ApJ, 512, L13

Mielke, E. W., \& Schunck, F. E. 2000, Nucl. Phys. B, 564, 185

Miralda-Escudé, J. 1998, ApJ, 501, L5

Mohapatra, R. N., \& Teplitz, V. L. 1999, Phys. Lett. B, 462, 302

Narayan, R., \& Yi, I. 1994, ApJ, 428, L13

Niemeyer, J. C., \& Jedamzik, K. 1999, Phys. Rev. D, 59, 124013

Peccei, R. D., \& Quinn, H. R. 1977, Phys. Rev. Lett., 38, 1440

Peebles, P. J. E., Seafer, S., \& Hu, W. 2000, ApJ, 539, L1

Quataert, E., \& Narayan, R. 1999, ApJ, 520, 298

Sahu, K. 1994, Nature, 370, 275

Schwarz, D. J., Schmid, C., \& Widerin, P. 1997, preprint (astro-ph/ 9704250)

Seljak, U., \& Zaldarriaga, M. 1996, ApJ, 469, 437

Silk, J. 1971, A\&A, 12, 421

Silk, J., Goldsmith, D. W., Field, G. B., \& Carrasco, L. 1972, A\&A, 20, 287

Silk, J., \& Werner, M. W. 1969, ApJ, 158, 185

Stone, J. M., Pringle, J. E., \& Begelman, M. C. 1999, MNRAS, 310, 1002

Tytler, D., O’Meara, J. M., Suzuki, N., \& Lubin, D. 2000, Phys. Scr., 85, 12

Yi, I., Narayan, R., Barrett, D., \& McClintock, J. E. 1996, A\&AS, 120, 187

Zaldarriaga, M., Spergel, D., \& Seljak, U. 1997, ApJ, 488, 1 